\title{
Paediatric emergency medicine point-of-care ultrasound: Fundamental or fad?
}

\author{
Peter SNELLING ${ }^{1,2,3}$ and Mark TESSARO ${ }_{\perp}^{4}$ \\ 1'Lady Cilento Children's Hospital, South Brisbane, Queensland, Australia, ${ }^{2}$ Greenslopes Private Hospital, Greenslopes, Queensland, Australia, \\ ${ }^{3}$ University of Queensland, Brisbane, Queensland, Australia, and ${ }^{4}$ Hospital for Sick Children, Toronto, Ontario, Canada
}

The traditional practice of medicine is increasingly challenged by disruptive technologies. One such tool in paediatric emergency medicine is point-of-care ultrasound (POCUS), now well established in North America but still emerging throughout Australasia. ${ }^{1,2}$ Like any new trend, the adoption of POCUS has both its advocates and sceptics given that a comprehensive ultrasound service is readily available in most departments. This article will examine both the current barriers and future directions of POCUS in paediatric emergency medicine.

\section{Stay focused}

POCUS arose from the simplification of ultrasound technology to a portable machine that can be used at the bedside. It comprises a rapid, focused assessment that is integrated into the clinical assessment of the patient without impeding, and potentially improving, work flow. ${ }^{3}$ These goaldirected ultrasounds glean both qualitative and quantitative information to answer a targeted clinical question, as opposed to a time-consuming, protocolised radiology-performed ultrasound. ${ }^{4}$ Its portability enables immediate use in the resuscitation environment to garner time-critical information. Conversely, the danger is that patients, caregivers or other medical professionals might feel that they have received a comprehensive diagnostic ultrasound when, actually, other broader findings might have been overlooked. POCUS does not obviate the need for comprehensive imaging but often places specific emphasis on the recognition of urgent and fixable problems. However, protracted scanning in EDs can become a source of consternation when the rest of the department is neglected. Nevertheless, as the cost and size of machines shrink, the technology is becoming more commonly used and might become a part of a doctor's armamentarium.

\section{Old dog, new tricks}

The learning curve of POCUS is steep, but studies have demonstrated rapid uptake and improvement of skills through didactic workshops and practice. ${ }^{4,5}$ POCUS is a complex skill that can be broken down into three main components: image acquisition, image interpretation and clinical integration. Whilst it varies with prior experience, generally, about 50 examinations are required to become a proficient user for a particular application. ${ }^{6}$ Becoming proficient might be considered an additional burden for those who are already overwhelmed or cognitively overloaded with competing educational priorities. Initiating training can be both intimidating and costly, with many not knowing where to begin given the paucity of resources within their department. Others see it as an opportunity to improve their delivery of patient care, diagnostic streaming and workplace efficiency and are willing to invest their time.

Correspondence: Dr Peter Snelling, Lady Cilento Children's Hospital, South Brisbane, QLD, Australia. Email: peter.j.snelling@gmail.com

60 Peter Snelling, BSc, MBBS (Hons), FRACP, Paediatrician.

\section{Operator please}

A significant barrier to the uptake of POCUS is the issue of operator dependence and credentialing. The indiscriminate use of ultrasound could lead to unnecessary investigations or false reassurance. ${ }^{3}$ Many argue that this is no different from other operator-dependent modalities, such as electrocardiogram interpretation, or the lack of requirement of being credentialed to use a stethoscope as a diagnostic tool. The tipping point of routine use of POCUS within a department is when there are proficient users who can then train others. This necessitates additional infrastructure for image storage, auditing and training, adding to already spiralling departmental cost. Cost-effective use has been demonstrated in the adult setting, and revenue might eventually be generated through billings for specific POCUS applications, as seen in North America. ${ }^{7}$ It is envisaged that POCUS training will soon become mandatory in North America, ${ }^{1,2}$ which might influence the Australasian context. ${ }^{8}$ Moreover, a recent review of litigation related to emergency POCUS in America revealed that lawsuits were related to failure to perform POCUS rather than interpretation failures when POCUS was utilised. ${ }^{9}$

\section{Cooperation makes it happen}

The emergence of POCUS within an institution can be disruptive, and opportunities for innovation and improved care can be lost to interdepartmental politics. Partnering with the Radiology Department ensures that their expertise benefits 
POCUS users and assures institutional leadership that appropriate stakeholders are engaged in the roll out of ultrasound use by novel operators. Many North American children's hospitals with longstanding POCUS programmes now have cross-disciplinary ultrasound committees that set clinical standards and foster collaboration in quality improvement and research. Creative solutions to hosting and sharing POCUS images across a hospital is key to these collaborations and allow findings to be reviewed both by POCUS experts and traditional ultrasound users in radiology or cardiology. Such image-sharing initiatives assist overtaxed radiology departments in accurately triaging consults and can expedite care when pathological findings obtained in one area of the hospital can be instantly reviewed in another. Image archiving and robust quality assurance review helps mitigate the risk of missed findings during the roll out of new POCUS programmes. Departments using POCUS should consider policies for quality assurance and patient contact in cases of possible discrepancies between initial findings and changes to these findings on expert review.

\section{Children are not small adults}

POCUS is ideal in the paediatric population given that it is non-invasive, that it does not emit ionising radiation and that a child's body habitus is generally ideal for imaging. ${ }^{3}$ POCUS is well established in adult emergency medicine, but not all adult applications can be translated to paediatrics. ${ }^{10}$ For example, there is ongoing discussion about the role of focused assessment with sonography in trauma (FAST) in paediatrics, where the clear majority of children with visceral injury are haemodynamically stable and are conservatively managed. ${ }^{11}$ Equally, there are paediatric applications, like intussusception and pyloric stenosis, that require specific training. ${ }^{12}$ However, the hand-eye coordination required for image acquisition and physics principles remain ubiquitous.

\section{It is not all black and white}

The original implementation of POCUS was as a binary decision tool, for example, a pneumothorax: lung sliding 'yes' or 'no'. However, this has become far more sophisticated, with POCUS now considered an extension of the physical examination that can augment clinical findings. ${ }^{13}$ Medical students are being taught anatomy via ultrasound rather than the traditional cadaveric dissection. ${ }^{14}$ This could eventually lead to physical examinations incorporating POCUS via handheld devices to definitively measure hepatomegaly or identify a ventricular septal defect, based on clinical findings raising the pretest probability. ${ }^{5,15,16}$ Case reports highlight that the familiarity with normal anatomy has led to the diagnosis of significant pathological findings. ${ }^{17}$ We have to keep in mind, however, that POCUS is unlikely to ever be the starting point of a patient's assessment-history taking and physical examination will remain key. Like any investigation, POCUS provides additional information that must be interpreted in the overall clinical context.

\section{Seeing is believing}

Although it does not negate the stethoscope, POCUS offers the additional advantage of allowing visualisation, rather than just auscultation, of internal structures. It is becoming standard practice for safeguarding needle-guided procedures, such as vascular line placement or regional nerve blockade. ${ }^{18,19}$ Simple applications, such as the identification of a hip effusion, can rapidly locate the reason for a child's limp but cannot differentiate transient synovitis from septic arthritis..$^{20}$ Constipation is a clinical diagnosis that can be supported by POCUS, providing objective feedback to parents whilst avoiding the temptation to conduct unnecessary radiographs. ${ }^{21,22}$ Global cardiac function, the filling of the inferior vena cava and the 'wetness' of the lungs can guide fluid resuscitation in shock, which can be difficult to discern from physiological parameters and clinical assessment alone in the paediatric population..$^{23}$ Measurement of the optic nerve sheath diameter is showing promise as a surrogate marker of increased intracranial pressure, but reference ranges are not well defined in the paediatric population. ${ }^{24}$ Although a rare life-threatening condition, echocardiography is the gold standard for the evaluation of cardiac tamponade, making this POCUS application compelling for departments with a limited cardiac service. ${ }^{25}$ The applications are numerous but vary in practicality and efficacy. ${ }^{26,27}$

\section{The future is in our hands}

The complete role of POCUS in paediatric emergency is still to be elucidated through evidence-based research. ${ }^{26}$ Lung ultrasound is an example of a growing field in which POCUS is showing enormous promise in children, being more diagnostically accurate for pneumonia than the standard chest radiograph, and might eventually differentiate aetiology. ${ }^{28,29}$ We are yet to realise how this increased accuracy translates into improved patient outcomes, such as avoiding sepsis or empyema, or whether it would lead to overtreatment. Realistically, it is unlikely to replace standard radiographs if inpatient teams are unable to monitor the disease progress via POCUS. Transoesophageal echocardiography during cardiopulmonary arrest might become standard practice as it enables direct visualisation the quality of cardiac compressions, can assess aetiology and limit interruptions during pulse checks. $^{30}$ However, paediatric cardiac arrests are infrequent with typically poor outcomes, and the additional training and resources required would be difficult to justify. Ultrasound-enhancing contrast might open up new possibilities not yet realised but might remain within the realms of dedicated sonographers and radiologists,

\section{Conclusion}

POCUS is a revolution that is challenging the traditional approach to 
1 assessing children in the ED. Whilst it is becoming standard practice to safeguard procedures, the benefits of its routine use for diagnostic assessments has yet to be validated but might have a defined role for augmenting clinical acumen. If indeed POCUS has the ability to improve patient outcomes, it behoves us to continue to explore and research its various applications.

\section{References}

1. American Academy of Pediatrics. Point-of-care ultrasonography by pediatric emergency medicine physicians. Pediatrics 2015; 135: e1113-22.

2. Rosenfield D, Kwan C, Fischer J. Point-of-care ultrasound: an emerging technology in Canadian paediatrics. Pediatr. Child Health 2015; 20: 67-8.

3. Moore CL, Copel JA. Point-of-care Ultrasonography. New England J. Med. 2011; 364: 749-57.

4. Carrie C, Biais M, Lafitte S, Grenier N, Revel P, Janvier G. Goal-directed ultrasound in emergency medicine: evaluation of a specific training program using an ultrasonic stethoscope. Eur. J. Emerg. Med. 2015; 22: 419-25.

5. Skalski JH, Elrashidi M, Reed DA, McDonald FS, Bhagra A. Using standardized patients to teach point-of-care ultrasound-guided physical examination skills to internal medicine residents. J. Grad. Med. Educ. 2015; 7: 95-7.

6. Blehar DJ, Barton B, Gaspari RJ. Learning curves in emergency ultrasound education. Acad. Emerg. Med. 2015; 22: 574-82.

7. Mitchell D, Gardiner AL, Vanderwerff L, Jones S, Thompson D, Leng P. Using pointof-care bedside ultrasound for volume assessment in early shock: an outcome study. Chest World Congr. 2016 https://doi.org/10. 1016/j.chest.2016.02.154.

8. Australasian College for Emergency Medicine, P21, 1999.

9. Stolz L, O’Brien KM, Miller ML, Winters-Brown ND, Blaivas $\mathrm{M}$, Adhikari S. A review of lawsuits related to point-of-care emergency ultrasound applications. Western J. Emerg. Med. 2015; 16: 1-4.
10. Whitson MR, Mayo PH. Ultrasonography in the emergency department. Crit. Care 2016; 20: 1-8.

11. Holmes JF, Kelley KM YXoottonGorges SL, Utter GH e, QD fffect of abdominal ultrasound on clinical care, outcomes, and resources use among children with blunt torso trauma: a randomized clinical trial. JAMA 2017; 317: 2290-6.

12. Gallagher RA, Levy JA. Advances in point-of-care ultrasound in pediatric emergency medicine. Curr. Opin. Pediatr. 2014; 26: 265-71.

13. Jauhar S. The demise of the physical exam. New England J. Med. 2006; 354: 548-51.

14. Rempell JS, $\mathrm{Ra}$ F, DiSalvo D, Kumar $\mathrm{N}$ et 2 ilot point-of-care ultrasound curriculum at Harvard Medical School: early experience. Western J. Emerg. Med. 2016; 17: 734-40.

15. Colli A, Pand, Fraquelli $\mathrm{M}$, Segato $S$ et $\Omega$ he use of a pocketsized ultrasound device improves physical examination: results of an inand outpatient cohort study. PLoS One 2015; 10: e0122181, 1-10.

16. Kobal SL, Trento L, Baharami S, Folstrup K, Naqvi TZ et T. lomparison of effectiveness 2 randcarried ultrasound to bedside cardiovascular physical examination. Am. J. Cardiol. 2005; 96: 1002-6.

17. Jamjoom RS, Etoom Y, Salano T, Desjardins M, Fischer JW. Emergency point-of-care ultrasound detection of cancer in the pediatric emergency department. Pediatr. Emerg. Care 2015; 31: 602-4.

18. National Institute for Heath and Care Excellent. Guidance on the use of ultrasound locating devices for placing central venous catheters. 2002 https://www.nice.org.uk/guidance/ta4 9/documents/appraisal-consultationdocument-ultrasound-locating-devi ces-for-placing-central-venous-lines

19. Baker M, Gullett JP. Ultrasoundguided femoral nerve blocks. Pediatr. Emerg. Care 2015; 31: 864-8.

20. Garrison J, Nguyen M, Marin JR. Emergency department point-ofcare hip ultrasound and its role in the diagnosis of septic hip arthritis: a case report. Pediatr. Emerg. Care 2016; 32: 555-7.
21. Doniger SJ, Dessie A, Latronica C. Measuring the transrectal diameter on point-of-care ultrasound to diagnose constipation in children. Pediatr. Emerg. Care 2016 10.1097/PEC.0000000000000775.

22. Pensabene L, Buonomo C, Fishman L, Chitkara D, Nurko S. Lack of utility of abdominal x-rays in the evaluation of children with constipation: comparison of different scoring methods. JPGN 2010; 51: $155-9$.

23. Modi P, Glavis-Bloom J, Nasrin S et al. Accuracy of inferior vena cava ultrasound for $\mathrm{p}$ ed ting dehydration in children with acute diarrhea in resource-limited settings. Plos One 2016; 11: e-0146859.

24. Le A, Hoehn ME, Smith ME, Spentzas T, Schlappy D, Pershad J. Bedside sonographic measurement of optic nerve sheath diameter as a predictor of increased intracranial pressure in children. Ann. Emerg. Med. 2009; 53: 785-91.

25. Smith AT, Watnick C, Ferre RM. Cardiac tamponade diagnosed by point-of-care ultrasound. Pediatr. Emerg. Care 2017; 33: 132-4.

26. Marin JR, Abo AM, Arroyo AC, Boniger SJ, Fischer Jw al. Pediatric emergency med point-ofcare ultrasound: summary of the evidence. Crit. Ultrasound J. 2016; 8: 1-83.

27. O'Brien AJ, Brady RM. Point-ofcare ultrasound in paediatric emergency medicine. J. Paediatr. Child Health 2016; 52: 174-80.

28. Pereda MA, Chavez MA, HooperMiele CC, Gilman RH Lung ultrasound for the di sis of pneumonia in children: a meta-analysis. Pediatrics 2015; 135: 714-22.

29. Varshney T, Mok E, Shapiro AJ, Li P, Dubrovsky AS. Point-of-care lung ultrasound in young children with respiratory tract infections and wheeze. Emerg. Med. J. 2016: 1-8.

30. Blaivas M. Tranesophageal echocardiography during cardiopulmonary arrest in the emergency department. Resuscitation 2008; 78: $135-40$

31. Barr R. How to develop a contrastenhanced ultrasound program. J. Ultrasound Med. 2017; 36: 1225-40. 